\title{
ABOUT THE ROLE OF THE FERMI-LIQUID EFFECTS ON THE ACOUSTIC TRANSPARENCY IN LAYERED CONDUCTORS
}

\author{
O. Galbova, G. Ivanovski, D. Krstovska \\ Institute of Physics, Faculty of Natural Sciences, P. O. Box 162 \\ Ss Cyril and Methodius University, Skopje, Macedonia
}

(Received April 3, 1998)

\begin{abstract}
Attenuation of the acoustic wave which is polarized and propagates along the normal to the layer of the quasi-two-dimensional conductor in sufficiently strong magnetic fields, when the electron radius is much smaller than the sound wavelength, i.e. $k r \ll 1$, is investigated theoretically. The electroacoustic coefficients strongly depend on the orientation of the magnetic field. For certain values of the angle between the direction of the magnetic field and a normal to the layer, an anomalous acoustic transparency occurs. The role of the Fermi-liquid interaction between conduction electrons on the acoustic transparency effect is studied.

Key words: quasi-two-dimensional conductor, acoustic wave, Fermi-liquid interaction, acoustic transparency.

PACS number(s): 72.15.Nj, 72.50.+b
\end{abstract}

In quasi-two-dimensional conductors, a number of specific effects take place due to a sharp anisotropy of charge carriers velocity $\mathbf{v}$ on the Fermi surface [1, 2].

An analysis of acoustic waves propagating in metals in a magnetic field resulted in successfully solving the inverse problem of reconstructing the electron energy spectrum from experimental data which was formulated by I. M. Lifshits. The concept of quasiparticles, viz., elementary excitations above the ground state of condensed media, is undoubtedly effective in the study of physical properties of various conductors, including lowdimensional ones.

Evidently, sharp anisotropy of the electrical conductivity is connected with the anisotropy of charge carriers velocity $\mathbf{v}=\partial \varepsilon / \partial \mathbf{p}$ on the Fermi surface $\varepsilon(\mathbf{p})=\varepsilon_{\mathrm{F}}$, i.e. their energy

$$
\varepsilon(\mathbf{p})=\sum_{n=0}^{\infty} \varepsilon_{n}\left(p_{x}, p_{y}\right) \cos \left(\frac{a n p_{z}}{\hbar}\right)
$$

is weakly dependent on the quasi-momentum projection $p_{z}=\mathbf{p n}$. The Fermi surface of such conductors is a mildly corrugated cylinder or a system of weakly corrugated cylinders and strongly stretched cavities in the momentum space.

Here, $a$ is the separation between the layers, $h=2 \pi \hbar$ is Planck's constant. The maximum value of the function $\varepsilon_{1}\left(p_{x}, p_{y}\right)$ on the Fermi surface is $\eta \varepsilon_{F} \ll \varepsilon_{F}$, and the maximum values of $\varepsilon_{n}\left(p_{x}, p_{y}\right)$ with $n \geq 2$ are even smaller.

The elementary excitations in the conductors form a Fermi liquid, and their energy spectrum is determined by the distribution function for quasiparticles. As a result, the response of the electron system in solids to an external perturbation depends essentially on the correlation functions which describe the electron-electron interaction.
The analysis of the galvanomagnetic phenomena when the charge carriers are assumed to form a Fermi-gas without concretizing the electron energy spectrum, is equivalent to the consideration of the problem in Fermiliquid theory.

The inclusion of the Fermi-liquid interaction of charge carriers leads to a renormalization of kinetic coefficients calculated under the assumption that conduction electrons form a Fermi gas.

The current interest in low-dimensional structures is mainly due to the need in new superconducting materials. However, the specific properties of such superconductors in the normal (nonsuperconducting) state can undoubtedly be used in various fields of acoustoelectronics.

In the case of a small deformation of crystal lattice, including the Fermi-liquid effects, the energy of elementary excitations has the form

$$
\varepsilon(\mathbf{p}, \mathbf{r}, t)=\varepsilon_{0}(\mathbf{p})+\lambda_{i j}(\mathbf{p}) u_{i j}+\Psi(\mathbf{p}, \mathbf{r}, t),
$$

where $\varepsilon_{0}(\mathbf{p})$ is the charge carriers energy in undeformed crystal in the gas approximation, $u_{i j}=\partial u_{i} / \partial x_{j}$ is the strain tensor of the crystal, $\mathbf{u}$ the displacement of ions, and $\lambda_{i j}(\mathbf{p})$ the deformation potential tensor. The last term in this formula takes into account the correlation effects associated with electron-electron interaction

$$
\Psi(\mathbf{p}, \mathbf{r}, t)=\frac{2}{(2 \pi \hbar)^{3}} \int \Phi\left(\mathbf{p}, \mathbf{p}^{\prime}\right) \delta f\left(\mathbf{p}^{\prime}, \mathbf{r}, t\right) d^{3} p
$$

Here $\delta f=f(\mathbf{p}, \mathbf{r}, t)-f_{0}\left(\varepsilon_{0}\right)$ is the nonequilibrium correction to the equilibrium Fermi distribution function $f_{0}\left(\varepsilon_{0}\right)$ for charge carriers in the undeformed conductor.

The charge carrier distribution function $f(\mathbf{p}, \mathbf{r}, t)$ required for calculating electroacoustic coefficients can be 
found from the kinetic Boltzmann equation

$\frac{\partial f}{\partial t}+\frac{\partial \varepsilon}{\partial \mathbf{p}} \frac{\partial f}{\partial \mathbf{r}}+\left[e \mathbf{E}-e \mu_{0}\left(\mathbf{H} \frac{\partial \varepsilon}{\partial \mathbf{p}}\right)-\frac{\partial \delta \varepsilon}{\partial \mathbf{r}}\right] \frac{\partial f}{\partial \mathbf{p}}=W_{c o l}\{f\}$,

where $W_{\text {col }}\{f\}$ is the collision integral which will be taken into account below in the $\tau$-approximation, i.e., $W_{c o l}\{f\}=\left[f_{0}(\varepsilon)-f\right] / \tau ; \tau=l / v$ is the mean free time for charge carriers, $\mathbf{v}$ their velocity, and $\mathbf{E}$ and $\mathbf{H}$ are the electric and magnetic field.

The equation of charge carriers motion in this case has the form

$$
\frac{d \mathbf{p}}{d t}=e \mathbf{E}+e \mu_{0}[\mathbf{v} \times \mathbf{H}]-\frac{\partial \delta \varepsilon}{\partial \mathbf{r}}
$$

where

$$
\delta \varepsilon=\left(\lambda_{i j}(\mathbf{p})-\widetilde{\lambda}_{i j}\right) u_{i j}+\Psi(\mathbf{p}, \mathbf{r}, t)-\widetilde{\Psi}(\mathbf{r}, t)
$$

is the renormalization of the charge carriers energy spectrum, and the bar over a symbol indicates the averaging over the Fermi surface

$$
\widetilde{g}=\langle g\rangle /\langle 1\rangle \text {. }
$$

We assume that the wave is monochromatic with the frequency $\omega$ so that the differentiation with respect to time is equivalent to the multiplication of the functions being differentiated by $-i \omega$.

In the linear approximation in a weak perturbation of conduction electrons under the action of deformation of the crystal the kinetic equation takes the form

$$
\frac{\partial \chi}{\partial t_{\mathrm{H}}}+\mathbf{v} \frac{\partial \chi}{\partial \mathbf{r}}+\hat{\nu} \chi=e \mathbf{v} \widetilde{\mathbf{E}}-i \omega \Lambda_{i j} u_{i j}-i \omega(\Psi-\widetilde{\Psi}),
$$

where the notations are

$$
\begin{gathered}
\widetilde{\mathbf{E}}=\mathbf{E}-i \omega \mu_{0}[\mathbf{u} \times \mathbf{H}]+\frac{m \omega^{2}}{e} \mathbf{u}, \quad \Lambda_{i j}(\mathbf{p})=\lambda_{i j}(\mathbf{p})-\widetilde{\lambda}_{i j}, \\
\hat{\nu}=\left(\frac{1}{\tau}-i \omega\right) ; \quad \frac{\partial}{\partial t_{\mathrm{H}}} \equiv e \mu_{0}[\mathbf{v} \times \mathbf{H}] \frac{\partial}{\partial \mathbf{p}} .
\end{gathered}
$$

Here $t_{\mathrm{H}}$ is the time of the charge carriers motion in the magnetic field.

Sound attenuation rate can be obtained by means of the solution of the elasticity theory equation for the ionic displacement $u$ :

$$
-\omega^{2} \varrho u_{i}=\lambda_{i j l m} \frac{\partial u_{l m}}{\partial x_{j}}+F_{i}
$$

where $\varrho$ and $\lambda_{i j l m}$ are density and elastic tensor of the crystal. The force exerted by the electrons on the vibrating lattice in the case of small deformations has the form

$$
F_{i}=\frac{\partial}{\partial x_{j}}\left\langle\Lambda_{i j} \chi\right\rangle+\mu_{0}[\mathbf{j} \times \mathbf{H}]_{i}+\frac{i \omega m}{e} j_{i}
$$

and the electric current density $j_{i}$ can be represented as follows:

$$
j_{i}=-\frac{2}{(2 \pi \hbar)^{3}} \int e v_{i} \chi \frac{\partial f_{0}}{\partial \varepsilon} d^{3} p \equiv\left\langle e v_{i} \chi\right\rangle .
$$

The electric field $\mathbf{E}$ accompanying the acoustic wave must be defined with the help of Maxwell equations

$$
\Delta \mathbf{E}+i \omega \mu_{0} \mathbf{j}=0
$$

and the electroneutrality condition for the conductor, which is equivalent to the continuity condition for the current, i.e.

$$
\operatorname{div} \mathbf{j}=0
$$

This system of equations in the most general form in the case of small deformations for an arbitrary energymomentum relation for charge carriers was obtained by Kontorovich [3].

Attenuation of longitudinally and transversally polarized acoustic wave which propagates in the layer plane, in the presence of external magnetic field when the radius of curvature of the charge carrier trajectory is much smaller than the mean free path, but considerably larger than the acoustic wavelength has been investigated theoretically $[4,5]$.

In the present work we investigate an acoustic wave which propagates along the normal to the layer of the quasi-two-dimensional conductor. We direct the $z$-axis along the wave vector $\mathbf{k}=\{0,0, k\}$. The solution of the kinetic equation in Fourier representation is given by

$$
\chi(k)=\hat{R}\left\{e \tilde{E}_{j}(k) v_{j}+k \omega \Lambda_{j z} u_{j}(k)-i \omega[\Psi(k, \mathbf{p})-\tilde{\Psi}(k)]\right\},
$$

where

$$
\hat{R} g=\int_{-\infty}^{t} d t_{1} g\left(t_{1}\right) \exp \left\{i k\left[z\left(t_{1}\right)-z(t)\right]+\hat{\nu}\left(t_{1}-t\right)\right\}
$$

is the resolvent of Eq. (8) which allows us to determine the function $\Psi(k, \mathbf{p})$ with the help of relation (3).

The function $\Psi(k, \mathbf{p}, t)$ satisfies the integral equation 


$$
\Psi(k, \mathbf{p}, t)=\left\langle\Phi\left(\mathbf{p}, \mathbf{p}^{\prime}\right)\left[\chi\left(k, \mathbf{p}^{\prime}, t\right)-i k \Lambda_{j z}\left(\mathbf{p}^{\prime}\right) u_{j}(k)-\Psi\left(k, \mathbf{p}^{\prime}, t\right)+\widetilde{\Psi}(k, t)\right]\right\rangle .
$$

The Landau correlation function $\Phi\left(\mathbf{p}, \mathbf{p}^{\prime}\right)$ can be expanded in the complete set of orthonormal functions $\phi_{n}(\mathbf{p})$

$$
\Phi\left(\mathbf{p}, \mathbf{p}^{\prime}\right)=\sum_{n=0}^{\infty} \Phi_{n} \phi_{n}(\mathbf{p}) \phi_{n}\left(\mathbf{p}^{\prime}\right)
$$

where

$$
\frac{2}{(2 \pi \hbar)^{3}} \int \phi_{n}(\mathbf{p}) \phi_{m}(\mathbf{p}) \delta\left(\varepsilon(\mathbf{p})-\varepsilon_{F}\right) d^{3} p=\left\langle\phi_{n}(\mathbf{p}) \phi_{m}(\mathbf{p})\right\rangle=\delta_{n m} .
$$

For brevity of computations only, we can limit ourselves to the first two functions $\phi_{n}(\mathbf{p})$ so that $\phi_{1}(-\mathbf{p})=\phi_{1}(\mathbf{p})$, $\phi_{2}(-\mathbf{p})=-\phi_{2}(\mathbf{p})$, while the remaining $\phi_{n}(\mathbf{p})$ with $n>2$ are equal to zero.

So, using equation (14) we obtain the following expression for the integral equation (15):

$$
\begin{aligned}
& \Psi(k, \mathbf{p})+\Phi_{1} \phi_{1}(\mathbf{p})\left\{\left\langle\phi_{1}(\mathbf{p}) \Psi(k, \mathbf{p})\right\rangle+i k\left\langle\phi_{1} \Lambda_{j z} u_{j}\right\rangle+i \omega\left\langle\phi_{1}(\mathbf{p}) \hat{R} \Psi(k, \mathbf{p})\right\rangle\right. \\
& \left.-\left\langle\phi_{1}(\mathbf{p}) \hat{R} e \mathbf{v} \widetilde{\mathbf{E}}(k)\right\rangle-k \omega\left\langle\phi_{1}(\mathbf{p}) \hat{R} \Lambda_{j z} u_{j}(k)\right\rangle\right\}+\Phi_{2} \phi_{2}(\mathbf{p})\left\{\left\langle\phi_{2}(\mathbf{p}) \Psi(k, \mathbf{p})\right\rangle\right. \\
& \left.+i \omega\left\langle\phi_{2}(\mathbf{p}) \hat{R} \Psi(k, \mathbf{p})\right\rangle-\left\langle\phi_{2}(\mathbf{p}) \hat{R} e \mathbf{v} \widetilde{\mathbf{E}}(k)\right\rangle-k \omega\left\langle\phi_{2}(\mathbf{p}) \hat{R} \Lambda_{j z} u_{j}(k)\right\rangle\right\}=0 .
\end{aligned}
$$

The solution of this equation can be represented as follows:

$$
\Psi(k, \mathbf{p})=e \widetilde{\mathbf{E}}(k)\left[\chi(k) \Phi_{1} \phi_{1}(\mathbf{p})+\boldsymbol{\xi}(k) \Phi_{2} \phi_{2}(\mathbf{p})\right]+k \omega u_{j}\left[\alpha_{j}(k) \Phi_{1} \phi_{1}(\mathbf{p})+\beta_{j}(k) \Phi_{2} \phi_{2}(\mathbf{p})\right]
$$

where the vectors $\boldsymbol{\chi}, \boldsymbol{\xi}$ and the scalars $\alpha_{j}, \beta_{j}$ are given by

$$
\begin{gathered}
\chi_{j}\left\{1+\Phi_{1}\left\langle\phi_{1}^{2}\right\rangle+i \omega \Phi_{1}\left\langle\phi_{1} \hat{R} \phi_{1}\right\rangle\right\}+i \omega \Phi_{2} \xi_{j}\left\langle\phi_{1} \hat{R} \phi_{2}\right\rangle=\left\langle\phi_{1} \hat{R} v_{j}\right\rangle, \\
i \omega \Phi_{1} \chi_{j}\left\langle\phi_{2} \hat{R} \phi_{1}\right\rangle+\xi_{j}\left\{1+\Phi_{2}\left\langle\phi_{2}^{2}\right\rangle+i \omega \Phi_{2}\left\langle\phi_{2} \hat{R} \phi_{2}\right\rangle\right\}=\left\langle\phi_{2} \hat{R} v_{j}\right\rangle, \\
\alpha_{j}\left\{1+\Phi_{1}\left\langle\phi_{1}^{2}\right\rangle+i \omega \Phi_{1}\left\langle\phi_{1} \hat{R} \phi_{1}\right\rangle\right\}+i \omega \beta_{j} \Phi_{2}\left\langle\phi_{1} \hat{R} \phi_{2}\right\rangle=\left\langle\phi_{1} \hat{R} \Lambda_{j z}\right\rangle-\frac{i}{\omega}\left\langle\phi_{1} \Lambda_{j z}\right\rangle, \\
i \omega \alpha_{j} \Phi_{1}\left\langle\phi_{2} \hat{R} \phi_{1}\right\rangle+\beta_{j}\left\{1+\Phi_{2}\left\langle\phi_{2}^{2}\right\rangle+i \omega \Phi_{2}\left\langle\phi_{2} \hat{R} \phi_{2}\right\rangle\right\}=\left\langle\phi_{2} \hat{R} \Lambda_{j z}\right\rangle .
\end{gathered}
$$

Using equations (14) and (18), it is convenient to represent the quantities $j_{i}(k)=\left\langle e v_{i} \chi(k)\right\rangle$ and $\left\langle\chi(k) \Lambda_{i z}\right\rangle$, which characterize the response of the electron system to the perturbation caused by the acoustic wave, in the form

$$
\begin{gathered}
j_{i}(k)=\sigma_{i j}(k) \widetilde{E}_{j}(k)+k \omega a_{i j}(k) u_{j}(k), \\
\left\langle\Lambda_{i z} \chi(k)\right\rangle=b_{i j}(k) \widetilde{E}_{j}(k)+k \omega c_{i j}(k) u_{j}(k),
\end{gathered}
$$

where the Fourier transforms of the electrical conductivity tensor $\sigma_{i j}(k)$ and electroacoustic tensor $a_{i j}(k), b_{i j}(k)$ and $c_{i j}(k)$ are described by the following expressions:

$$
\sigma_{i j}(k)=e^{2}\left\{\left\langle v_{i} \hat{R} v_{j}\right\rangle-i \omega \Phi_{1}\left\langle v_{i} \hat{R} \phi_{1}\right\rangle \chi_{j}-i \omega \Phi_{2}\left\langle v_{i} \hat{R} \phi_{2}\right\rangle \xi_{j}\right\},
$$




$$
\begin{gathered}
a_{i j}(k)=e\left\{\left\langle v_{i} \hat{R} \Lambda_{j z}\right\rangle-i \omega \Phi_{1}\left\langle v_{i} \hat{R} \phi_{1}\right\rangle \alpha_{j}-i \omega \Phi_{2}\left\langle v_{i} \hat{R} \phi_{2}\right\rangle \beta_{j}\right\}, \\
b_{i j}(k)=e\left\{\left\langle\Lambda_{i z} \hat{R} v_{j}\right\rangle-i \omega \Phi_{1}\left\langle\Lambda_{i z} \hat{R} \phi_{1}\right\rangle \chi_{j}-i \omega \Phi_{2}\left\langle\Lambda_{i z} \hat{R} \phi_{2}\right\rangle \xi_{j}\right\}, \\
c_{i j}(k)=\left\{\left\langle\Lambda_{i z} \hat{R} \Lambda_{j z}\right\rangle-i \omega \Phi_{1}\left\langle\Lambda_{i z} \hat{R} \phi_{1}\right\rangle \alpha_{j}-i \omega \Phi_{2}\left\langle\Lambda_{i z} \hat{R} \phi_{2}\right\rangle \beta_{j}\right\} .
\end{gathered}
$$

For $k r \ll 1$ electroacoustic coefficients strongly depend on the orientation of the magnetic field and retaining only the first term in the expansion in $k r \ll 1$ of the resolvent

$$
\hat{R}_{0} g(t) \simeq \int_{-\infty}^{t} d t^{\prime} g\left(t^{\prime}\right) e^{\widehat{\nu}\left(t-t^{\prime}\right)}
$$

one can easily see that the functions $\chi_{j}$ and $\beta_{j}$ are equal to zero, and

$$
\begin{gathered}
\xi_{j}=\left\langle\phi_{2} \hat{R}_{0} v_{j}\right\rangle\left\{1+\Phi_{2}\left\langle\phi_{2}^{2}\right\rangle+i \omega \Phi_{2}\left\langle\phi_{2} \hat{R}_{0} \phi_{2}\right\rangle\right\}^{-1} \\
\alpha_{j}=\left[\left\langle\phi_{1} \hat{R}_{0} \Lambda_{j z}\right\rangle-\frac{i}{\omega}\left\langle\phi_{1} \Lambda_{j z}\right\rangle\right]\left\{1+\Phi_{1}\left\langle\phi_{1}^{2}\right\rangle+i \omega \Phi_{1}\left\langle\phi_{1} \hat{R}_{0} \phi_{1}\right\rangle\right\}^{-1} .
\end{gathered}
$$

In the main approximation in the small parameters $k r \ll 1$ and $\gamma \equiv \hat{\nu} / \Omega \ll 1$, electroacoustic coefficients $\sigma_{i j}(0)$ and $c_{i j}(0)$ take the form

$$
\begin{gathered}
\sigma_{i j}(0)=e^{2} \frac{1}{\nu}\left\langle\bar{v}_{i} \bar{v}_{j}\right\rangle-e^{2} \frac{i \omega}{\nu^{2}} \Phi_{2}\left\langle\bar{v}_{i} \bar{\phi}_{2}\right\rangle\left\langle\bar{\phi}_{2} \bar{v}_{j}\right\rangle\left\{1+\Phi_{2}+\frac{i \omega}{\nu} \Phi_{2}\left\langle\bar{\phi}_{2}^{2}\right\rangle\right\}^{-1}, \\
c_{i j}(0)=\frac{1}{\nu}\left\langle\bar{\Lambda}_{i z} \bar{\Lambda}_{j z}\right\rangle-\frac{i \omega}{\nu} \Phi_{1}\left\langle\bar{\Lambda}_{i z} \bar{\phi}_{1}\right\rangle\left[\frac{1}{\nu}\left\langle\bar{\phi}_{1} \bar{\Lambda}_{j z}\right\rangle-\frac{i}{\omega}\left\langle\bar{\phi}_{1} \Lambda_{j z}\right\rangle\right]\left\{1+\Phi_{1}+\frac{i \omega}{\nu} \Phi_{1}\left\langle\bar{\phi}_{1}^{2}\right\rangle\right\}^{-1},
\end{gathered}
$$

where

$$
\bar{g}=\frac{1}{T} \int_{0}^{T} d t g(t) .
$$

The magnetic field $\mathbf{H}=\{0, H \sin \theta, H \cos \theta\}$ is perpendicular to $x$-axis so that $\bar{v}_{x}=0$ and the components of the electrical conductivity tensor with one or both indices $x$ are equal to zero.

The dispersion equation for an acoustic transversally polarized wave propagating along the normal to the layer in a magnetic field $\mathbf{H}=\{0, H \sin \theta, H \cos \theta\}$ assumes the form

$$
\begin{gathered}
\left(\tilde{\sigma}_{y y}(0)-\frac{k^{2}}{i \omega \mu_{0}}\right)\left[\omega^{2} \varrho-k^{2} \varrho s_{\ell}^{2}+i k^{2} \omega c_{z z}(0)\right]=0, \\
s_{\ell}^{2}=\frac{\lambda_{z z z z}}{\varrho}, \quad \tilde{\sigma}_{\alpha \beta}=\sigma_{\alpha \beta}-\sigma_{\alpha z} \frac{\sigma_{z \beta}}{\sigma_{z z}}
\end{gathered}
$$

which excludes the interaction between the electromagnetic and acoustic waves.

One of the roots of the dispersion equation (25) close to $\omega / s_{\ell}$ determines the attenuation length for the acoustic wave and the renormalization of its velocity due to the interaction with conduction electrons. Such renormalizations are normally insignificant, and the asymptotic expression for $k_{1}$ for small $k_{1}=k-\omega / s_{\ell}$ has the form

$$
k_{1} \simeq \frac{i k^{2}}{2 \varrho s_{\ell}} c_{z z}(0)_{\mid k=\frac{\omega}{s_{\ell}}}
$$

The electroacoustic coefficient $c_{z z}(0)$ has been calculated in the case of the simplest energy-momentum relation for charge carriers in an unperturbed crystal, i.e.,

$$
\varepsilon(\mathbf{p})=\frac{p_{x}^{2}+p_{y}^{2}}{2 m^{*}}+\eta \frac{\hbar}{a} v_{0} \cos \frac{a p_{z}}{\hbar}
$$


and taking into account that the magnetic field is in $y z$ plane so that

$$
p_{z}=\frac{p_{\mathrm{H}}}{\cos \theta}-p_{y} \tan \theta .
$$

When $\theta=\theta_{c}$ the electroacoustic coefficient $c_{z z}(0)$ goes to zero, and for $\tan \theta \gg 1$ these zeros are repeated with the period $\Delta(\tan \theta)=\frac{2 \pi \hbar}{D_{p}}$, where $D_{p}$ is the extremal diameter of the Fermi surface along the axis $p_{y}$. The condition $\tan \theta \gg 1$ allows calculations to be done by using the stationary phase method.
We also assume for brevity, that each section of the Fermi surface by the plane $p_{\mathrm{H}}=$ const contains only two stationary-phase points at which $\mathbf{k v}\left(t_{1,2}\right)=\omega$.

To an accuracy to terms of the order $\eta^{2}$ we obtain the following expression for $k_{1}$

$$
k_{1}=\frac{i \omega^{2}}{2 \varrho s_{\ell}^{3}} \frac{\eta^{2} L^{2} \hbar}{\pi a \nu \mathcal{D}_{p} \tan \theta}\left[1+\sin \left(a \frac{\mathcal{D}_{p} \tan \theta}{\hbar}\right)\right]\{\langle 1\rangle-\mathcal{L}\}
$$

where the term

$$
\mathcal{L}=\frac{2 \Phi_{1}}{1+\Phi_{1}+\frac{i \omega}{\nu} \Phi_{1}\left\langle\bar{\phi}_{1}^{2}\right\rangle}\left\langle\bar{\phi}_{1} \cos \frac{a p_{\mathrm{H}}}{\hbar \cos \theta}\right\rangle\left[\left\langle\phi_{1}\left(t_{1}\right) \cos \frac{a p_{\mathrm{H}}}{\hbar \cos \theta}\right\rangle+\frac{i \omega}{\nu}\left\langle\bar{\phi}_{1} \cos \frac{a p_{\mathrm{H}}}{\hbar \cos \theta}\right\rangle\right]
$$

determines the contribution of Fermi-liquid interaction between the charge carriers.

The imaginary part of the root of the dispersion equation $k_{1}$ determines the coefficient of absorption of acoustic energy in the conductor and the real part describes the renormalization of the velocity of sound.

The inclusion of the next term which is linear in the small parameter $k r \ll 1$ gives only a small correction in $k_{1}$.

The result (29) shows that the attenuation length of the acoustic wave polarized along the normal is $\eta^{-2}$ times that for the wave polarized in the layer plane [5].

One can easily see that in strong magnetic fields when the electron path between two points of a stationary phase changes by half the wavelength, i.e.,

$$
\frac{a \mathcal{D}_{p} \tan \theta}{\hbar}=\pi\left(2 n-\frac{1}{2}\right)
$$

the conductor becomes transparent for the acoustic wave.

In that case the next terms in the expansions in the small parameters $k r, \gamma \ll 1$ should be taken into account and the absorption coefficient for the acoustic wave obtains the form

$$
\Gamma_{t r a .}=\eta^{2} \Gamma_{0}\left[a_{1} \gamma^{2}+a_{2}(k r)^{2}\right]
$$

where $a_{1}$ and $a_{2}$ are functions of $\phi_{1}$ and $\phi_{2}$ respectively, and $\Gamma_{0}$ coincides in order of magnitude with the absorption coefficient in zero magnetic field.

The maxima of the absorption coefficient are determined with the conditions

$$
\frac{a \mathcal{D}_{p} \tan \theta}{\hbar}=\pi\left(2 n+\frac{1}{2}\right)
$$

The conditions of maximum attenuation of acoustic wave energy as well as the conditions of acoustic transparency are periodically repeated which allows to determine the diameter $D_{p}$ of the Fermi surface.

The inclusion of the Fermi-liquid interaction of charge carriers has a significant influence on the form of the resonance curves. This interaction does not destroy the conditions for the maximum attenuation of the sound energy, as well as those for acoustic transparency.

\section{ACKNOWLEDGMENT}

We would like to thank V. G. Peschansky and O. V. Kirichenko for their helpful discussions. We are also grateful to the Ministry of Science of Republic of Macedonia for financial assistance to the project within the framework of which this research was carried out.
[1] V. M. Gohfel'd, O. V. Kirichenko, V. G. Peschansky, Zh. Eksp. Teor. Fiz. 108, 2147 (1995).

[2] O. V. Kirichenko, V. G. Peschansky, J. Phys. (Paris) 4, 823 (1994).

[3] V. M. Kontorovich, Usp. Fiz. Nauk. 142, 266 (1984).
[4] O. Galbova, G. Ivanovski, O. V. Kirichenko, V. G. Peschansky, Fiz. Nizk. Temp. 23, 173 (1997).

[5] O. Galbova, G. Ivanovski, O. V. Kirichenko, V. G. Peschansky, Fiz. Nizk. Temp. 22, 425 (1996). 